STRUCTURAL BIOLOGY COMMUNICATIONS

ISSN 2053-230X

\section{Crystal structure of the Agrobacterium tumefaciens type VI effector-immunity complex}

\author{
Satoshi Fukuhara, Takanori Nakane, Keitaro Yamashita, Ryohei Ishii, \\ Ryuichiro Ishitani* and Osamu Nureki*
}

Department of Biological Sciences, Graduate School of Science, The University of Tokyo, 7-3-1 Hongo, Bunkyo-ku,
Tokyo 113-0033, Japan. *Correspondence e-mail: ishitani@bs.s.u-tokyo.ac.jp, nureki@bs.s.u-tokyo.ac.jp

Received 10 October 2018

Accepted 18 November 2018

Edited by I. Tanaka, Hokkaido University, Japan

Keywords: Agrobacterium tumefaciens; type VI effector-immunity complex; crystal structure; Tae4; Tai4.

PDB references: Tai4 dimer, 6ije; Tae4-Tai4 complex, 6ijf

Supporting information: this article has supporting information at journals.iucr.org/f

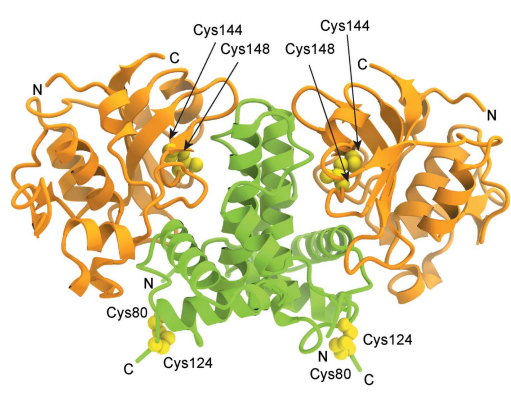

OPEN $\odot$ ACCESS
The type VI secretion system (T6SS) comprises needle-shaped multisubunit complexes that play a role in the microbial defense systems of Gram-negative bacteria. Some Gram-negative bacteria harboring a T6SS deliver toxic effector proteins into the cytoplasm or periplasm of competing bacteria in order to lyse and kill them. To avoid self-cell disruption, these bacteria have cognate immunity proteins that inhibit their toxic effector proteins. T6SS amidase effector protein 4 (Tae4) and T6SS amidase immunity protein 4 (Tai4) are a representative of the toxic effector-immunity pairs of the T6SS. Here, the threedimensional structures of Tai4 and the Tae4-Tai4 complex from Agrobacterium tumefaciens are reported at 1.55 and $1.9 \AA$ A resolution, respectively. A structural comparison with other Tae4-Tai4 homologs revealed similarities and differences in the catalytic and inhibitory mechanisms among the Tae4 and Tai4 family proteins.

\section{Introduction}

The type VI secretion systems (T6SSs) of Gram-negative bacteria inject various toxic effectors into the periplasmic or cytoplasmic space of the target cells and induce cell lysis of enemy cells (Hood et al., 2010; MacIntyre et al., 2010; Schwarz et al., 2010; Murdoch et al., 2011; Russell et al., 2011, 2012). The various T6SS-related amidase effector proteins (Taes) are classified into four families (Tae1, Tae2, Tae3 and Tae4) based on their cleavage specificities (Russell et al., 2012). These effectors and the unique bacterial secretion system, T6SS, which responds to enemy bacteria, enable Gram-negative bacteria to attack targeted heterologous cells (Russell et al., 2012). In addition to these toxic effectors, Gram-negative bacteria have four amidase immunity proteins (Tai1, Tai2, Tai3 and Tai4). Tai1, Tai2, Tai3 and Tai4 neutralize the endogenous toxic effectors Tae1, Tae2, Tae 3 and Tae4, respectively. These effector-immunity pairs (Tae1-Tai1, Tae2-Tai2, Tae3-Tai3 and Tae4-Tai4) generally originate from the same operons. The presence of cognate effector-immunity pairs suggests that self-protection systems with the co-expression of effector proteins and immunity proteins are a common feature in Gram-negative bacteria possessing a T6SS (Russell et al., 2012).

Tae4-Tai4 is the fourth T6SS-related effector-immunity pair to be structurally determined. Previous studies reported the structures of the Tae4-Tai4 complexes from Enterobacter cloacae, Salmonella typhimurium and Serratia marcescens (Zhang, Gao et al., 2013; Zhang, Zhang et al., 2013; Benz et al., 2013; Srikannathasan et al., 2013). Comparisons of these structures revealed that $S$. marcescens Tai4 is structurally different from E. cloacae Tai4 and S. typhimurium Tai4, 
whereas the Tae 4 proteins from the different species are highly conserved (Srikannathasan et al., 2013). However, the crystal structures of Tae4-Tai4 complexes from other species have remained unknown.

To gain insight into the Tae 4 family proteins, we determined the crystal structures of Tai4 and of the Tae4-Tai4 complex from Agrobacterium tumefaciens at 1.55 and $1.9 \AA$ resolution, respectively.

\section{Materials and methods}

\subsection{Macromolecule production}

The genes encoding the Tai4 and Tae4 proteins from A. tumefaciens (ATU4346 and ATU4347, respectively) were codon-optimized for Escherichia coli and synthesized by Invitrogen. The SignalP 4.1 server (Petersen et al., 2011) was used to predict the signal peptide of $A$. tumefaciens Tai4 (AtTai4). The AtTai4 gene segment (residues 26-129) without the putative signal sequence was PCR-amplified and inserted into the pCold-GST vector. The plasmid was transformed into E. coli Rosetta 2 (DE3) cells for protein expression.

The cells were grown in Luria-Bertani (LB) medium at $310 \mathrm{~K}$ until the $\mathrm{OD}_{600}$ reached 0.8 ; gene expression was then induced with $0.5 \mathrm{~m} M$ isopropyl $\beta$-D-1-thiogalactopyranoside (IPTG) following a reduction in the temperature to $277 \mathrm{~K}$. Cell growth was continued for $24 \mathrm{~h}$ at $288 \mathrm{~K}$. The $\mathrm{N}$-terminally $\mathrm{His}_{6}$-GST-tagged AtTai4 was affinity-purified using an $\mathrm{Ni}-$ NTA column (Qiagen). The N-terminal $\mathrm{His}_{6}$-GST tag was removed by incubation with Turbo3C protease (Nacalai Tesque) for $16 \mathrm{~h}$ at $277 \mathrm{~K}$. After rechromatography on the Ni-NTA column, further purification was conducted by ionexchange chromatography on a Resource Q column (GE Healthcare) and gel-filtration chromatography on a HiLoad Superdex 75 column (GE Healthcare). The purified samples were concentrated to $8.6 \mathrm{mg} \mathrm{ml}^{-1}$ for crystallization.

For co-expression of the AtTae4-AtTai4 complex, the AtTai4 gene segment (residues 26-129) was cloned into the first multiple cloning site of the pETDuet-1 vector (Novagen) and the $A t \mathrm{Tae} 4$ gene segment (residues 1-163) was subsequently cloned into the second multiple cloning site. A Tobacco etch virus (TEV) protease-recognition sequence was introduced between the $\mathrm{His}_{6}$ tag and the AtTai4 sequence by a PCR-based method. The plasmid was transformed into E. coli Rosetta 2 (DE3) cells for overexpression. The cells were cultured in LB medium at $310 \mathrm{~K}$ until the $\mathrm{OD}_{600}$ reached 0.8 ; gene expression was then induced with $0.5 \mathrm{~m} M$ IPTG following a temperature reduction to $277 \mathrm{~K}$. The cells were further cultured at $291 \mathrm{~K}$ for $24 \mathrm{~h}$. The AtTae4-AtTai4 complex was affinity-purified using an Ni-NTA column (Qiagen). The N-terminal $\mathrm{His}_{6}$ tag was removed by incubation with TEV protease for $24 \mathrm{~h}$ at $277 \mathrm{~K}$. After rechromatography on the Ni-NTA column, the complex was further purified by ion-exchange chromatography on a Resource $\mathrm{Q}$ column and subsequent gel-filtration chromatography on a HiLoad Superdex 75 column. The purified complex was concentrated to $13 \mathrm{mg} \mathrm{ml}^{-1}$ for crystallization trials.
Table 1

Data-collection and refinement statistics.

Values in parentheses are for the outer shell.

\begin{tabular}{lll}
\hline & & \\
& AtTai4 & At Tae4-AtTai4 \\
complex
\end{tabular}

\subsection{Crystallization}

Initial crystallization trials were performed at $293 \mathrm{~K}$ by the sitting-drop vapor-diffusion method in a 96-well crystallization plate using various commercially available screening kits. Crystallization drops were prepared by mixing $200 \mathrm{nl}$ purified protein solution and $200 \mathrm{nl}$ reservoir solution using a Mosquito crystallization robot (TTP Labtech). The initial crystals of AtTai4 were optimized at $293 \mathrm{~K}$ by varying the concentrations of PEG and salt in the reservoir solution using an Additive Screen kit (Hampton Research). Plate-shaped crystals of AtTai4 were obtained in 33\% PEG 6000, $1.5 \mathrm{M}$ lithium chloride, $100 \mathrm{~m} M$ sodium acetate. The AtTae4-AtTai4 complex formed thick plate-shaped crystals using MemGold reservoir condition E11 consisting of 35\% PEG 400, $0.05 M$ Tris $\mathrm{pH}$ 8.5, $0.05 M$ sodium sulfate, $0.05 M$ lithium sulfate.

\subsection{Data collection and processing}

All crystals were cryoprotected in reservoir solution supplemented with $25 \%$ ethylene glycol and flash-cooled in a 
nitrogen-gas stream. X-ray diffraction data for AtTai4 and for the AtTae4-AtTai4 complex were collected on beamlines BL41XU and BL32XU at SPring-8, Hyogo, Japan using a PILATUS3 6M detector (Dectris) and an MX225HS detector (Rayonix), respectively. The continuous helical data-collection scheme was applied using $12 \times 8 \mu \mathrm{m}($ AtTai4 $)$ and $18 \times 1 \mu \mathrm{m}$ (AtTae4-AtTai4 complex) beams. Diffraction data were integrated with DIALS (Waterman et al., 2016) and scaled with AIMLESS (Evans \& Murshudov, 2013). The data-collection statistics are shown in Table 1.

\subsection{Structure determination}

The structures of AtTai4 and the AtTae4-AtTai4 complex were solved by molecular replacement with MOLREP (Vagin \& Teplyakov, 2010) using the structures of Tai4 from S. marcescens (PDB entry 3zfi; Srikannathasan et al., 2013) and the Tae4-Tai4 complex from $S$. marcescens (PDB entry 4bi8; Srikannathasan et al., 2013), respectively, as search models. Model building and structure refinement were performed
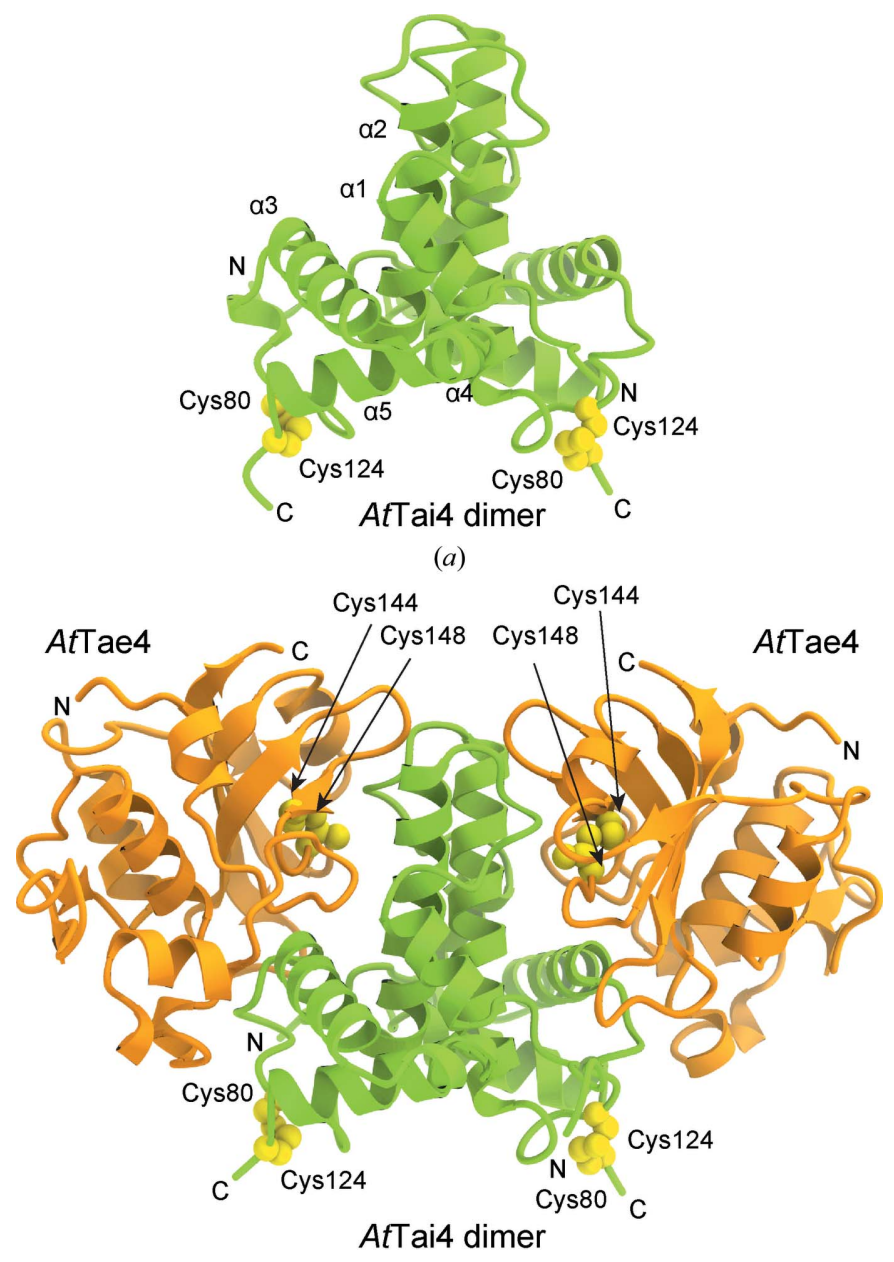

Figure 1

(b)

Crystal structures of the AtTai4 homodimer and the AtTae4-AtTai4 complex. (a) Structural overview of the AtTai4 homodimer. The intramolecular disulfide bonds between Cys 80 and Cys 124 are shown as yellow spheres. ( $b$ ) Overall structure of the $A t$ Tae4-AtTai4 complex. The intramolecular disulfide bonds between Cys144 and Cys148 are shown as yellow spheres. using Coot (Emsley et al., 2010) and REFMAC5 (Murshudov et al., 2011), respectively. Ramachandran plot analysis was performed using MolProbity (Chen et al., 2010). The refinement statistics are shown in Table 1. The atomic coordinates and structure factors of AtTai4 and the AtTae4-AtTai4 complex have been deposited in the Protein Data Bank (PDB) with accession codes 6ije and 6ijf, respectively. X-ray diffraction images have been also deposited in the Zenodo data repository (https://doi.org/10.5281/zenodo.1453302).

\section{Results and discussion}

\subsection{Overall structure}

The crystal structure of AtTai4 was determined at $1.55 \AA$ resolution. AtTai4 forms a homodimer composed of five $\alpha$-helices ( $\alpha 1-\alpha 5$; Fig. 1a). The $\alpha 2$ helix (residues 52-74) contributes to dimer formation in the asymmetric unit, which is consistent with the size-exclusion chromatography results indicating that $A t$ Tai4 exists as a dimer in solution. A disulfide bond is formed between Cys 80 and Cys124 in each protomer (Fig. 1a). In addition, we determined the crystal structure of the AtTae4-At Tai4 complex at $1.9 \AA$ resolution (Fig. 1b). The structure revealed that the AtTai4 dimer binds two AtTae4 molecules to form a heterotetramer in the asymmetric unit, which is consistent with the size-exclusion chromatography results indicating that the AtTae4-AtTai4 complex exists in a heterotetrameric form in solution. The crystal structure revealed that $A t$ Tae4 forms an intramolecular disulfide bond between Cys144 and Cys148, which may confer structural stability (Fig. $1 b$ ). Superimposition of AtTai4 alone and AtTai4 bound to $A t$ Tae 4 resulted in a root-mean-square deviation (r.m.s.d.) value of $0.8 \AA$, indicating that no structural changes occur upon complex formation.

\subsection{Structure comparison}

A search for structural homologs was conducted using the DALI server (Holm \& Laakso, 2016). The top-scoring structural homolog of AtTai4 was the Rap1a protein from S. marcescens (SmTai4; PDB entry 3zfi; Srikannathasan et al., 2013), with a $Z$-score of 13.5 and an r.m.s.d. of $1.7 \AA$. The structural homologs of AtTae4 are the following proteins: the Ssp1 protein from $S$. marcescens (SmTae4; PDB entry 4bi3; Srikannathasan et al., 2013), the Tae4 protein from E. cloacae (EcTae4; PDB entry 4hfk; Zhang, Zhang et al., 2013) and the Tae4 protein from S. typhimurium (StTae4; PDB entry 4j30; Benz et al., 2013). The most similar structural homolog was the $S m$ Tae4 protein, with a $Z$-score of 25.4 and an r.m.s.d. of $1.6 \AA$. Amino-acid sequence alignments of $A t$ Tai4 and AtTae4 with their homologs are shown in Figs. 2(a) and 2(b). At Tai4 shares $32.3 \%$ amino-acid sequence identity with $\operatorname{SmTai4}$. At Tae4 shares $41.5 \%, 21.9 \%$ and $20.5 \%$ sequence identity with SmTae4, StTae4 and EcTae4, respectively. Therefore, the At Tae4-AtTai4 complex structure deepens our understanding of the structurally distinct interactions of the Tae4 and Tai4 family proteins. 
A comparison of AtTai4 with $S m$ Tai4 revealed that $A t$ Tai4 contains a longer $\alpha 2$ helix and a longer loop between the $\alpha 1$ and $\alpha 2$ helices (Fig. 3a). As the longer $\alpha 2$ helix and loop between the $\alpha 1$ and $\alpha 2$ helices interact with two At Tae4 molecules in the asymmetric unit (Fig. 1b), AtTae4 and AtTai4 have structurally distinct interactions compared with the SmTae4-SmTai4 complex. In addition, neither the StTae4$S t$ Tai4 complex nor the EcTae4-EcTai4 complex has these interactions. Glu53 and Arg56 in the $\alpha 2$ helix form hydrogen bonds to Gln143 and Ser18 in one of the AtTae 4 molecules in the asymmetric unit, respectively (Fig. 3b). Pro47, Asp48 and
Val49 in the loop of AtTai4 form hydrogen bonds to Arg108, Thr142 and Arg108 in the other AtTae4 protomer in the asymmetric unit, respectively. In addition, Ser50 in the loop of At Tai4 interacts with Ser139 and Glu140 in AtTae4 (Fig. $3 b$ ). Thus, these specific interactions between $A t$ Tae 4 and $A t$ Tai4 may contribute towards stabilizing the formation of the AtTae4-At Tai4 complex.

The structure of the SmTae4-SmTai4 complex revealed that $S m$ Tai4 is located at the entrance to the active site of SmTae4, where it blocks substrate access to the active site (Srikannathasan et al., 2013). The catalytic Gln84 of SmTai4 forms a

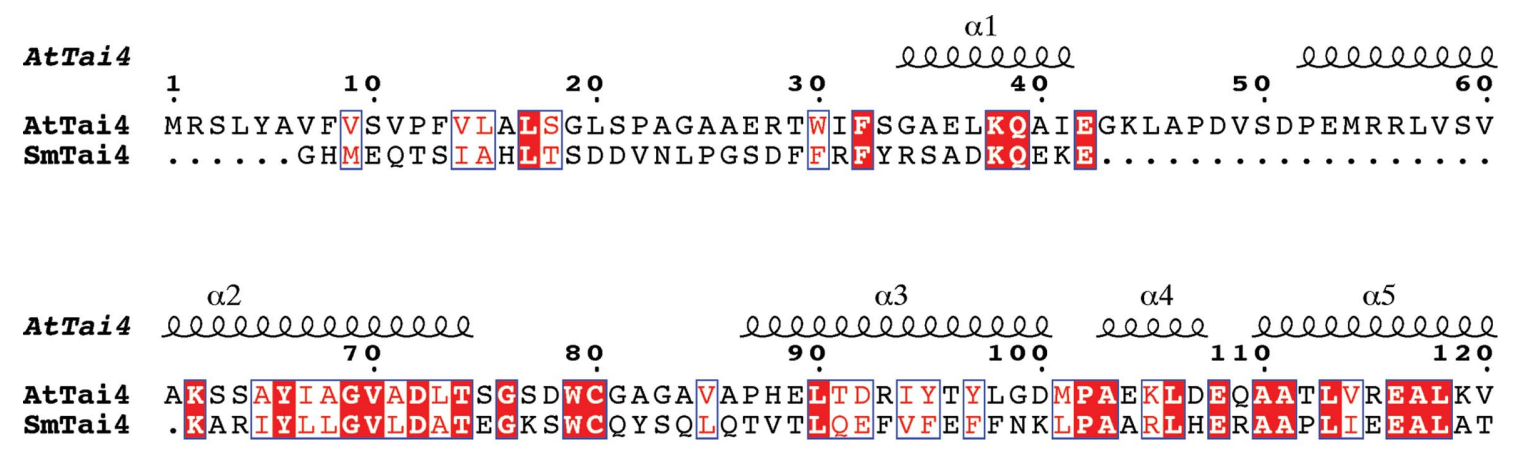

$\begin{array}{ll}\text { AtTai4 } & \ell \\ & \\ \text { AtTai4 } & \text { SFPCEQKSN } \\ \text { SmTai4 } & \text { RFPCKGGKA }\end{array}$

(a)

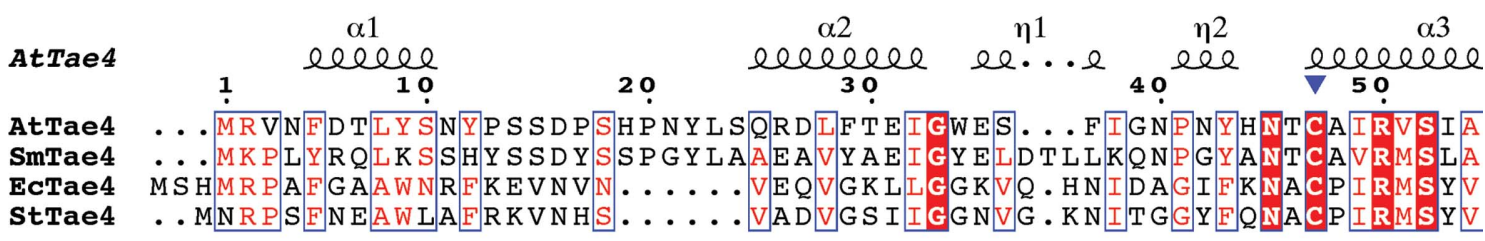

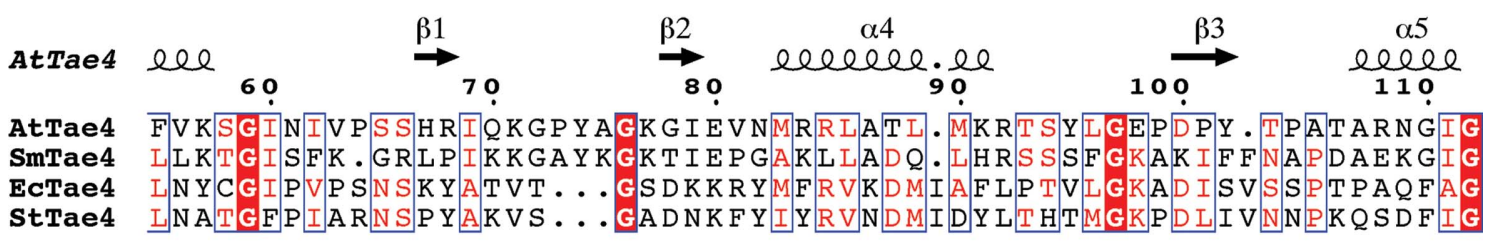

AtTae4

AtTae4

SmTae4

EcTae4

StTae4
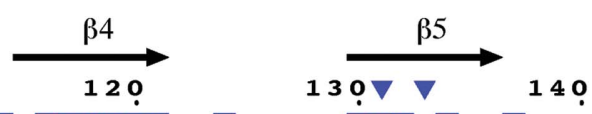

$\stackrel{\beta 6}{\longrightarrow}$

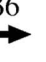

KKGVVFFNKITNYD . GGHIDLIEPENSLLTCHSHC . . . Y Y F NKEVWFWELS . . .

. KQGI I I T GHGWLDATGHVTLWNGN I CSDDCHFIGSPGNGSFIPTNATFWSLK . . .

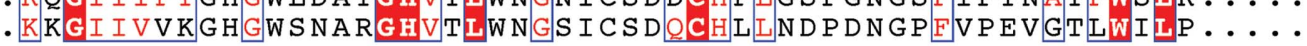

(b)

Figure 2

Structure-based sequence alignments of AtTai4 and AtTae4 with their homologs performed with Clustal Omega and ESPript3. (a) Sequence alignment of At Tai4 with SmTai4 (PDB entry 3zfi; Srikannathasan et al., 2013). (b) Sequence alignment of AtTae4 with SmTae4 (PDB entry 4 bi3; Srikannathasan et al., 2013), EcTae4 (PDB entry 4hfk; Zhang, Zhang et al., 2013) and StTae4 (PDB entry 4j30; Benz et al., 2013). The potential catalytic triad residues, Cys47, His131 and Asp133, are indicated by blue triangles. 


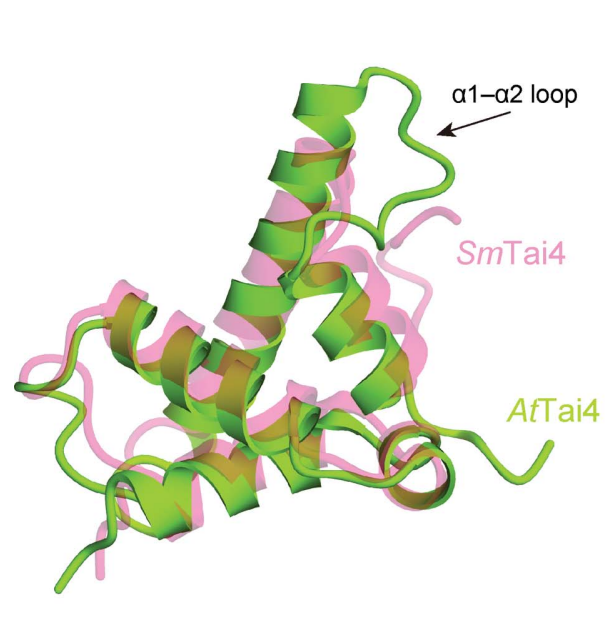

(a)

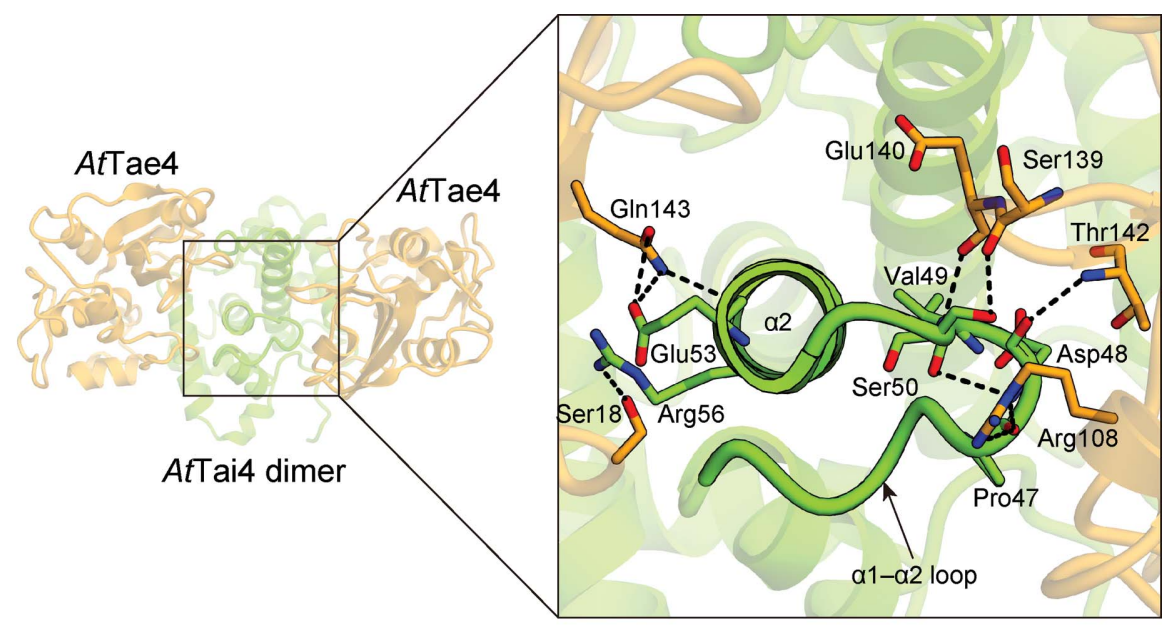

(b)

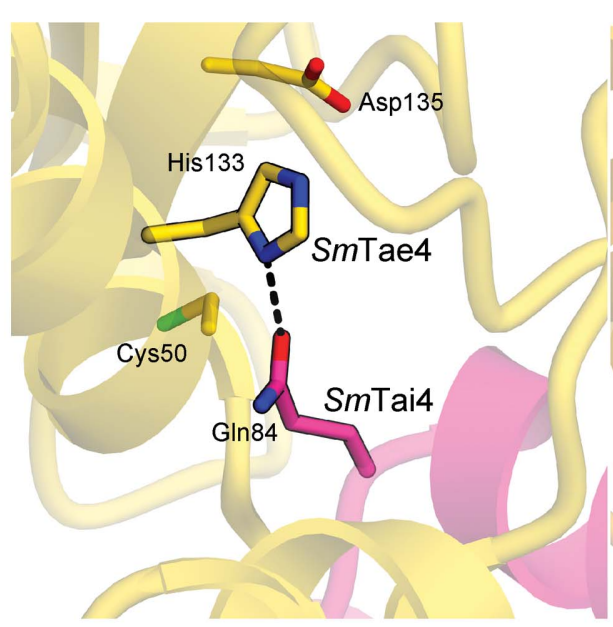

(c)

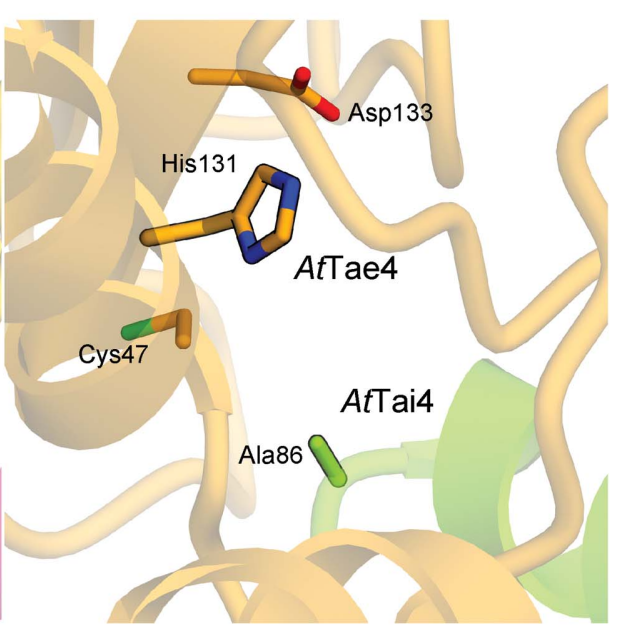

(d)

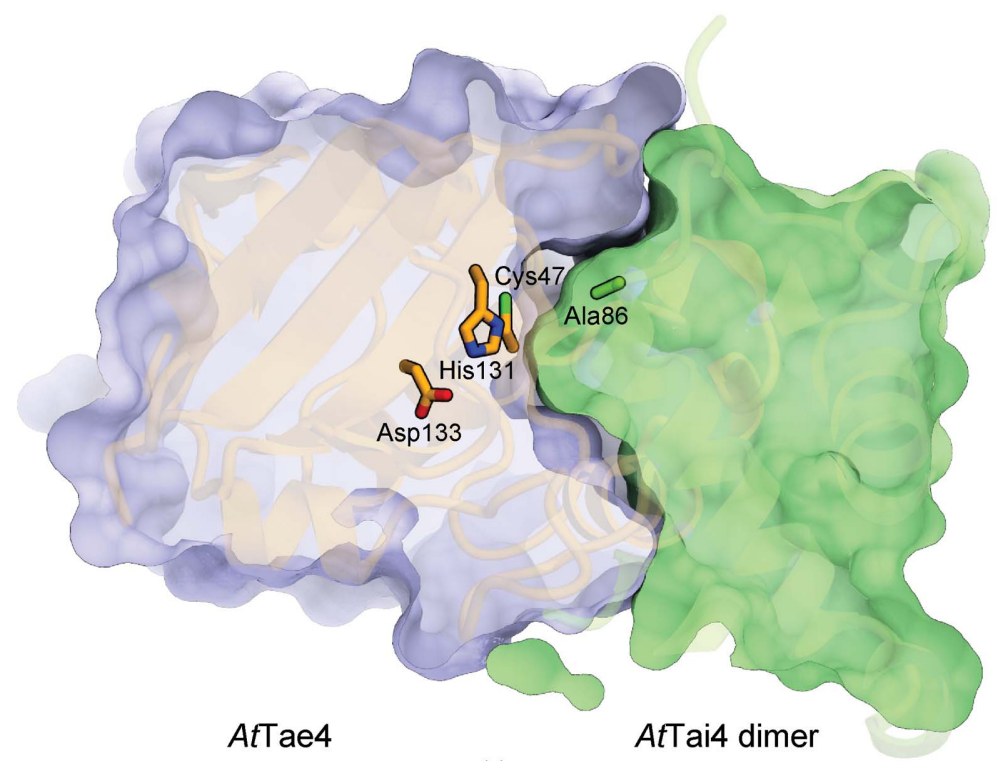

(e)

Figure 3

Structural comparison of the AtTae4-AtTai4 complex with the SmTae4-SmTai4 complex. (a) A superimposition of AtTai4 and $S m$ Tai4 indicated that At Tai 4 contains extensions in the $\alpha 2$ helix and in the loop between the $\alpha 1$ and $\alpha 2$ helices. (b) The residues involved in the interaction between $A t$ Tai 4 and $A t$ Tae4. (c) The crystal structure of the SmTae4-SmTai4 complex revealed that Gln84 of SmTai4 interacts with His 133 of $S m$ Tae4 and blocks the active site (PDB entry 4bi8; Srikannathasan et al., 2013). (d) The crystal structure of the AtTae4-AtTai4 complex lacks the interaction between the expected catalytic His131 of AtTae4 and the corresponding residue of AtTai4. The glutamine is not conserved in AtTai4 and is replaced by an alanine in AtTai4. (e) The AtTai4 homodimer is positioned close to the AtTae4 active-site surface and may block substrate binding. 
hydrogen bond to His133 of SmTae4 and blocks the active site (Fig. 3c; Srikannathasan et al., 2013). Amino-acid sequence alignment of SmTai4 and AtTai4 demonstrated that Gln84 of $S m$ Tai4 is not conserved and is replaced by Ala86 in AtTai4 (Fig. 2a). In the present structure of the AtTae4-AtTai4 complex, although the potentially catalytic His131 residue of At Tae4 does not interact with any residues of AtTai4 (Fig. 3d), At Tai4 blocks the entrance to the substrate-binding pocket of AtTae4 and prevents substrate access to the active site (Fig. 3e).

Previous studies have suggested that AtTai4 and SmTai4 neutralize the activities of $A t$ Tae 4 and $S m$ Tae4, respectively. The morphological abnormality mediated by $S m$ Tae 4 was neutralized by SmTai4 (Srikannathasan et al., 2013). The growth inhibition of $E$. coli $\mathrm{DH} 10 \mathrm{~B}$ owing to the expression of AtTae4 was rescued by the co-expression of AtTai4 (Ma et al., 2014). While there is no direct interaction between the potentially catalytic His131 of AtTae4 and Ala86 of AtTai4, which corresponds to Gln84 of SmTai4, the structural comparison suggests that AtTai4 effectively neutralizes the activity of AtTae4 by blocking the entrance to its substratebinding pocket.

\subsection{Catalytic site}

The Tae4 family proteins have conserved catalytic residues (Cys-His-Asp) that are responsible for their peptidoglycan amidase activity. Cys46, His128 and Asp139 of EcTae4 and Cys44, His126 and Asp137 of StTae4 form the catalytic triads, which are similar to the canonical catalytic triad in the papainlike cysteine peptidase (PDB entry 1bp4; LaLonde et al., 1998) (Zhang, Gao et al., 2013; Zhang, Zhang et al., 2013; Benz et al., 2013; Fig. 4a). SmTae4 also has a catalytic triad formed by Cys50, His133 and Asp135. While Asp139 of EcTae4 and Asp137 of StTae4 are replaced by Ser148 in SmTae4, Asp135 of SmTae4, which corresponds to Thr130 of EcTae4 and Thr128 of StTae4, is located at a position similar to those of Asp139 of EcTae4 and Asp137 of StTae4 in the SmTae4

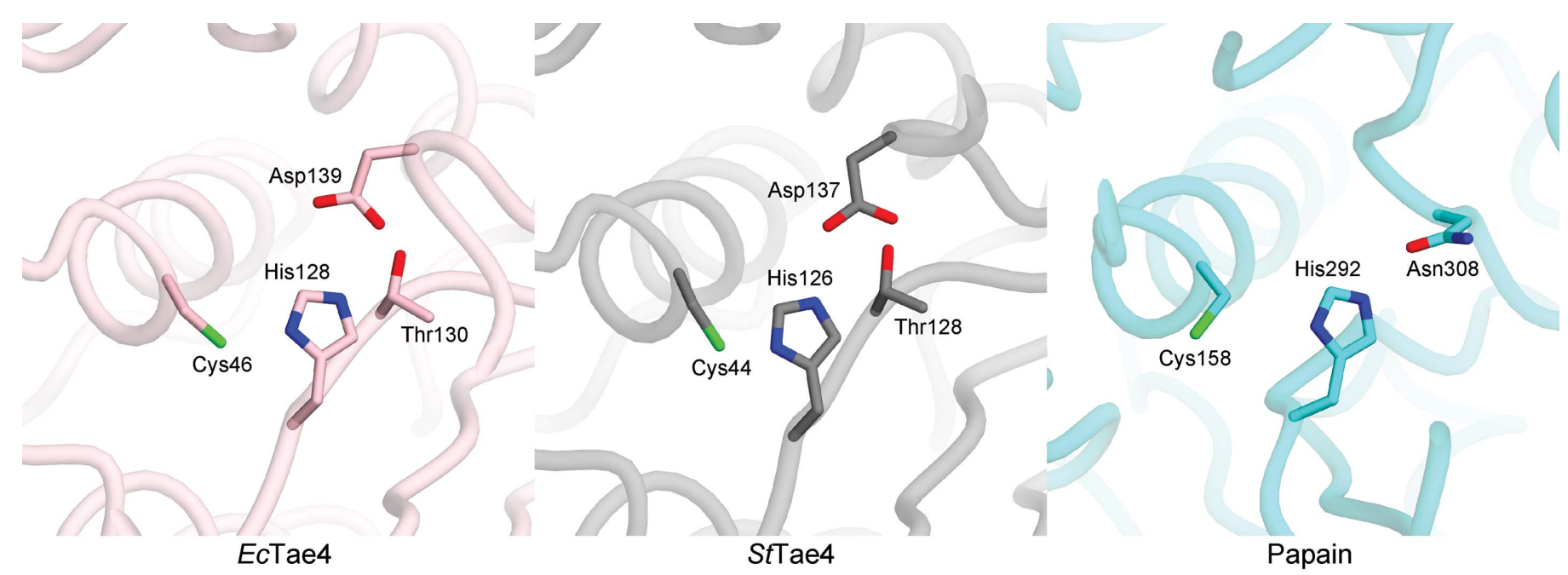

(a)

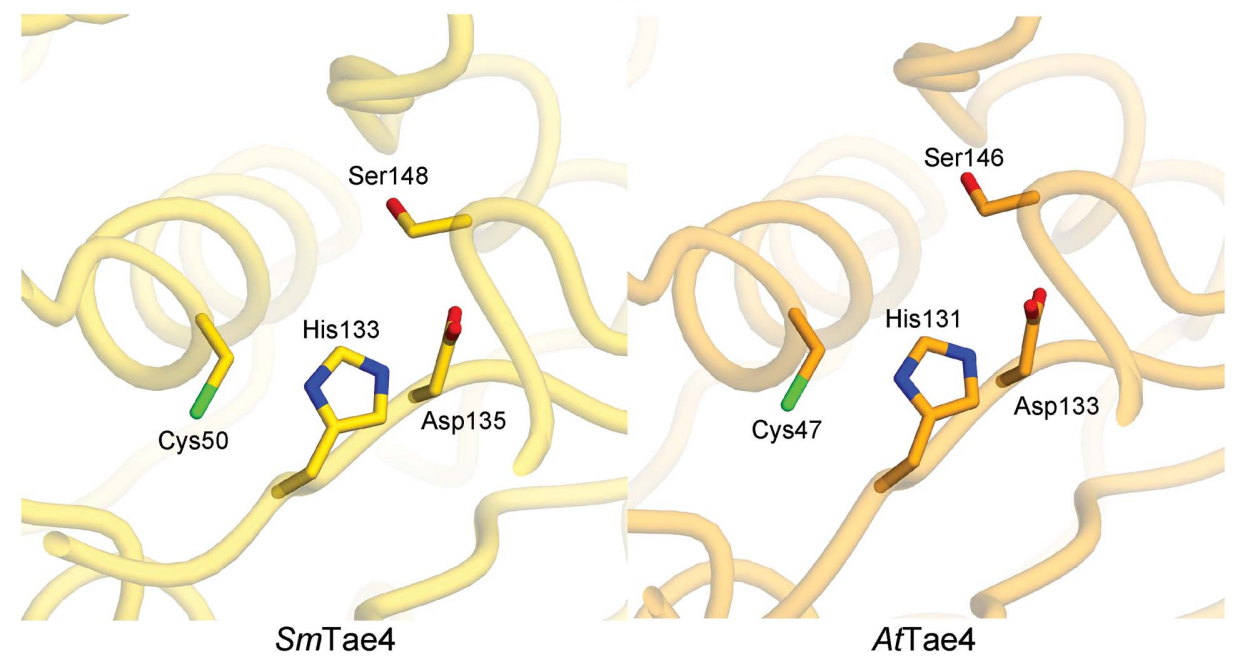

(b)

Figure 4

Structural differences in the catalytic triad. (a) EcTae4 and StTae4 have a conserved catalytic active center containing the catalytic residues (Cys-HisAsp), which have a similar arrangement to the catalytic triad of papain (PDB entry 1bp4; LaLonde et al., 1998). (b) SmTae4 and AtTae4 have the conserved catalytic residues (Cys-His-Asp), but the third aspartic acid in the catalytic triad has a distinct spatial arrangement. 
structure (Figs. $4 a$ and $4 b$ ). These observations suggested that Asp135 serves as the third aspartic acid residue in the catalytic triad in SmTae4 (Srikannathasan et al., 2013). In the present structure of the AtTae4-AtTai4 complex, Cys47, His131 and Asp133 also form a catalytic triad, as in the SmTae4-SmTai4 complex structure (Fig. 4b). Thus, the present structure reinforces the idea that the Tae4 family proteins have two types of structurally distinct catalytic triads.

\section{Conclusion}

In this work, we determined the crystal structures of AtTai4 and the AtTae4-AtTai4 complex. Comparisons of these structures with those of homologous proteins revealed that the AtTae4-AtTai4 complex shares structural similarity with the SmTae4-SmTai4 complex. A structural comparison of AtTai4 with $S m$ Tai4 showed that AtTai4 contains more extended helices and loops, which may enforce the interaction between AtTai4 and the adjacent AtTae4. A structural superimposition highlighted the differences in the spatial arrangement of the aspartic acid residue in the catalytic triad (Cys-His-Asp) among the Tae4 family proteins. The present structures enhance our understanding of the catalytic and inhibitory mechanisms of the Tae4 and Tai4 family proteins.

\section{Acknowledgements}

We thank the beamline staff at BL41XU and BL32XU of SPring-8, Hyogo, Japan for support during data collection and Dr Hiroshi Nishimasu for critical comments, valuable suggestions and encouragement.

\section{Funding information}

This work was supported by a grant from the Core Research for Evolutional Science and Technology Program, the Creation of Basic Chronic Inflammation, from the Japan Science and Technology Agency to ON.

\section{References}

Benz, J., Reinstein, J. \& Meinhart, A. (2013). PLoS One, 8, e67362. Chen, V. B., Arendall, W. B., Headd, J. J., Keedy, D. A., Immormino, R. M., Kapral, G. J., Murray, L. W., Richardson, J. S. \& Richardson, D. C. (2010). Acta Cryst. D66, 12-21.

Emsley, P., Lohkamp, B., Scott, W. G. \& Cowtan, K. (2010). Acta Cryst. D66, 486-501.

Evans, P. R. \& Murshudov, G. N. (2013). Acta Cryst. D69, 1204-1214.

Holm, L. \& Laakso, L. M. (2016). Nucleic Acids Res. 44, W351-W355.

Hood, R. D., Singh, P., Hsu, F., Güvener, T., Carl, M. A., Trinidad, R. R. S., Silverman, J. M., Ohlson, B. B., Hicks, K. G., Plemel, R. L., Li, M., Schwarz, S., Wang, W. Y., Merz, A. J., Goodlett, D. R. \& Mougous, J. D. (2010). Cell Host Microbe, 7, 25-37.

LaLonde, J. M., Zhao, B., Smith, W. W., Janson, C. A., DesJarlais, R. L., Tomaszek, T. A., Carr, T. J., Thompson, S. K., Oh, H.-J., Yamashita, D. S., Veber, D. F. \& Abdel-Meguid, S. S. (1998). J. Med. Chem. 41, 4567-4576.

Ma, L.-S., Hachani, A., Lin, J.-S., Filloux, A. \& Lai, E.-M. (2014). Cell Host Microbe, 16, 94-104.

MacIntyre, D. L., Miyata, S. T., Kitaoka, M. \& Pukatzki, S. (2010). Proc. Natl Acad. Sci. USA, 107, 19520-19524.

Murdoch, S. L., Trunk, K., English, G., Fritsch, M. J., Pourkarimi, E. \& Coulthurst, S. J. (2011). J. Bacteriol. 193, 6057-6069.

Murshudov, G. N., Skubák, P., Lebedev, A. A., Pannu, N. S., Steiner, R. A., Nicholls, R. A., Winn, M. D., Long, F. \& Vagin, A. A. (2011). Acta Cryst. D67, 355-367.

Petersen, T. N., Brunak, S., von Heijne, G. \& Nielsen, H. (2011). Nature Methods, 8, 785-786.

Russell, A. B., Hood, R. D., Bui, N. K., Leroux, M., Vollmer, W. \& Mougous, J. D. (2011). Nature (London), 475, 343-347.

Russell, A. B., Singh, P., Brittnacher, M., Bui, N. K., Hood, R. D., Carl, M. A., Agnello, D. M., Schwarz, S., Goodlett, D. R., Vollmer, W. \& Mougous, J. D. (2012). Cell Host Microbe, 11, 538-549.

Schwarz, S., Hood, R. D. \& Mougous, J. D. (2010). Trends Microbiol. 18, 531-537.

Srikannathasan, V., English, G., Bui, N. K., Trunk, K., O'Rourke, P. E. F., Rao, V. A., Vollmer, W., Coulthurst, S. J. \& Hunter, W. N. (2013). Acta Cryst. D69, 2468-2482.

Vagin, A. \& Teplyakov, A. (2010). Acta Cryst. D66, 22-25.

Waterman, D. G., Winter, G., Gildea, R. J., Parkhurst, J. M., Brewster, A. S., Sauter, N. K. \& Evans, G. (2016). Acta Cryst. D72, 558-575.

Zhang, H., Gao, Z.-Q., Wei, Y., Xu, J.-H. \& Dong, Y.-H. (2013). PLoS One, 8, e73782.

Zhang, H., Zhang, H., Gao, Z.-Q., Wang, W.-J., Liu, G.-F., Xu, J.-H., Su, X.-D. \& Dong, Y.-H. (2013). J. Biol. Chem. 288, 5928-5939. 